\title{
Encadeamentos produtivos e multiplicadores macrossetoriais no Brasil após a abertura comercial
}

\author{
Productive chains and macro-sector multipliers in Brazil \\ after trade opening \\ José Firmino de Sousa Filho, Gervásio Ferreira dos Santos e \\ Luiz Carlos de Santana Ribeiro**
}

\begin{abstract}
Resumo: Este artigo analisa o processo de desenvolvimento produtivo da economia brasileira de 1990 a 2015 aplicando os índices de encadeamento produtivo e os multiplicadores setoriais para a produção e emprego. A pesquisa pretende contribuir para as discussões acerca das temáticas relativas ao crescimento macrossetorial da economia brasileira explorando o processo de crescimento setorial como uma ferramenta essencial das relações de produção, demanda e crescimento tecnológico. O banco de dados é composto pelas matrizes de Insumo-Produto (MIP's) elaboradas pelo IBGE nos períodos de 1990-1995-2000-2005-2010-2015, com 12 setores agregados. As matrizes foram utilizadas para calcular os índices Rasmussen-Hirschman, índice de ligações puras GHS e os multiplicadores clássicos de produção e emprego. Os resultados apontam que a estrutura produtiva da economia brasileira não evoluiu na direção de criação e desenvolvimento de encadeamentos produtivos consolidados. Além disso, os multiplicadores de produção e de emprego diminuíram em grande parte dos setores considerados.
\end{abstract}

Palavras-chave: Economia Brasileira. Insumo-Produto. Encadeamentos produtivos. Multiplicadores setoriais

\begin{abstract}
This paper analyzes the productive development process of the Brazilian economy from 1990 to 2015 by applying indexes of productive chaining and sectoral multipliers for production and employment. The research intends to contribute to the discussions about the themes related to the macro-sectorial growth of the Brazilian economy by exploring the sectorial growth process as an essential tool for the relations of production, demand, and technological growth. The database is composed of the InputOutput matrices (MIP's) prepared by IBGE in the periods 1990-1995-2000-2005-2010-

\footnotetext{
* Submissão: 06/04/2020 | Aprovação: 10/03/2021 | DOI: 10.5380/re.v42i79.72694

** Respectivamente: (1) Doutorando em Economia Aplicada pela Universidade Federal da Bahia (PPGE/UFBA) | ORCID: 0000-0001-5057-385X | E-mail: jose.sousa@ ufba.br | (2) Professor do Programa de Pós-Graduação em Economia da Universidade Federal da Bahia (PPGE/UFBA) | ORCID: 0000-00023545-3590 |E-mail: gervasios@ufba.br | (3) Professor e Pesquisador do Laboratório de Economia Aplicada e Desenvolvimento Regional da Universidade Federal de Sergipe (LEADER/UFS) | ORCID: 0000-00016374-3811 | E-mail: ribeiro.luiz84@gmail.com | Esta pesquisa teve apoio da Fundação de Amparo à Pesquisa do Estado da Bahia (FAPESB) e do Conselho Nacional de Desenvolvimento científico e Tecnológico (CNPq).
} 
2015, with 12 aggregated sectors. The matrices were used to calculate the RasmussenHirschman indices, GHS pure linkages index and the classical production and employment multipliers. The results indicate that the production structure of the Brazilian economy has not evolved in the direction of creation and development of consolidated productive chains. Furthermore, the production and employment multipliers have decreased in most of the sectors considered.

Keywords: Brazilian economy. Input-Output. Production. Productive chains. Sectorial multipliers

JEL: L11. O10. O14 


\section{Introdução}

A abertura comercial brasileira teve consequências heterogêneas sobre a estrutura produtiva nacional. Notadamente, a difusão de novos produtos, por meio da ampliação dos mercados comerciais, promoveu alterações na demanda e no nível de preços da economia. A substituição dos insumos produtivos pode ser gradual ou ser um fator de um processo revolucionário, no qual, a depender dos recursos tecnológicos envolvidos, promove alterações na dinâmica do mercado de trabalho e na estrutura econômica como um todo (Pasinetti, 1993). O aumento da variedade de novos produtos no mercado permite que as indústrias disponham de mudanças tecnológicas para seu crescimento por meio de termos qualitativos, o que induz a novas formas e processos coevolutivos relacionados.

A década de 1990 foi um período de mudanças e transformações estruturais para a economia. O Brasil era caracterizado como uma economia fechada para o resto do mundo, com a maior parte do consumo final e intermediário fornecidos por empresas domésticas e, de modo geral, de baixa qualidade e diversidade; as exportações também representavam uma pequena parcela da demanda final (Moreira et al., 2008). Nesse período, a economia brasileira abriu-se de forma acentuada, gerando grandes oportunidades, mas também mostrando gargalos produtivos e estruturais. Cabe ressaltar que a década de 1990 foi marcada por baixas taxas de crescimento do PIB, planos de estabilização sem sucesso e mudanças de moedas, até chegar em 1994, com o Plano Real.

A análise do crescimento setorial brasileiro a partir de 1990 é importante para se verificar como os setores evoluíram ao longo no tempo no que se refere à capacidade de encadeamentos e multiplicadores produtivos. Nesse sentido, Dietzenbacher (1992), Wolff e Nadiri (1993), Guilhoto e Picerno (1995), Dietzenbacher (2000), Guilhoto et al. (2005), Dietzenbacher (2005), Guilhoto (2010), Timmer et al. (2012), Harada (2015) e Rodrigues et al. (2016) ressaltam a relevância da mensuração dos linkage effects e multiplicadores setoriais para estabelecer quais são os setores-chave da economia, ou seja, os setores capazes de gerar maiores encadeamentos produtivos e spillovers.

Assim, a análise da estrutura de produção setorial baseada no método de Insumo-Produto é importante para verificar a capacidade setorial de promover crescimento econômico a partir das conexões traçadas entre a indústria, a agropecuária e os serviços com seus subsetores econômicos. Os efeitos diretos 
dessas relações impactam o aumento da produção, emprego, valor adicionado, impostos e exportações, ao passo que os efeitos indiretos são sentidos de acordo com as conexões traçadas entre os setores (Guilhoto; Sesso Filho, 2005).

Portanto, o objetivo desta pesquisa é analisar o processo de desenvolvimento produtivo da economia brasileira de 1990 a 2015 aplicando os índices de encadeamentos produtivos e multiplicadores setoriais. Desse modo, será possível contribuir com as discussões sobre o fortalecimento da estrutura produtiva para a manutenção do crescimento econômico sustentado. Para tanto, serão realizados os cálculos dos multiplicadores da produção e do emprego, o índice de Rasmussen-Hirschman e o índice de ligações puras GHS. Além disso, é importante ressaltar o papel de políticas macrossetoriais capazes de determinar estratégias de desenvolvimento que vão no sentido da criação de emprego e renda e, desse modo, geram um círculo virtuoso em toda a economia.

Por fim, a pesquisa divide-se em mais quatro seções além desta introdução. Na seção 2 é feita uma revisão sobre o processo de abertura comercial e a trajetória do crescimento setorial mais recente; na seção 3 é apresentada a base de dados e a metodologia. Na seção 4 são apresentados e discutidos os resultados e, por fim, são feitas as considerações finais.

\section{Abertura comercial brasileira e composição da estrutura produtiva}

A abertura comercial brasileira intensificou a necessidade de reestruturação dos setores produtivos, pois houve aumento da concorrência externa. Para tanto, privatizações de diversos segmentos da infraestrutura nacional, assim como setores industriais e extrativistas, entraram em pauta. Em 1999, o Estado promoveu reformas estruturais a fim de estimular a produção industrial e atrair mais investimentos externos para o país, dentre essas reformas, destacaram-se o regime de câmbio flutuante, as metas de inflação e de superávit primário.

As privatizações e as desregulamentações provocaram reestruturações produtivas na propriedade do capital e na produtividade com a finalidade de induzir o crescimento econômico sustentado. Porém, a taxa de crescimento da economia brasileira na década de 1990 foi menor que a média de crescimento da década anterior, conhecida como a "década perdida". Na década de 1980, a média de crescimento da economia brasileira foi de 3,0\%, já em 1990 foi de 1,8\% (Giambiagi; Moreira, 1999). 
A partir dos anos 2000, período marcado por crescimento econômico com estabilidade monetária, a estrutura de participação setorial no PIB não se alterou de forma significativa. Apenas no setor de comércio a participação no PIB aumentou de forma considerável entre 2002 e 2018, saindo de aproximadamente $7 \%$ para $11 \%$. Nos setores de serviços, a administração pública e outros serviços continuaram sendo os com maior representatividade, em torno de $15 \%$ e $16 \%$, respectivamente, em 2018. No que se refere aos setores industriais, a indústria de transformação e a construção civil foram os setores mais relevantes na participação do PIB, em torno de $9,7 \%$ e $3,8 \%$, respectivamente, conforme se pode observar nas figuras 1 e 2 .

Figura 1 - Participação dos setores de serviços no PIB

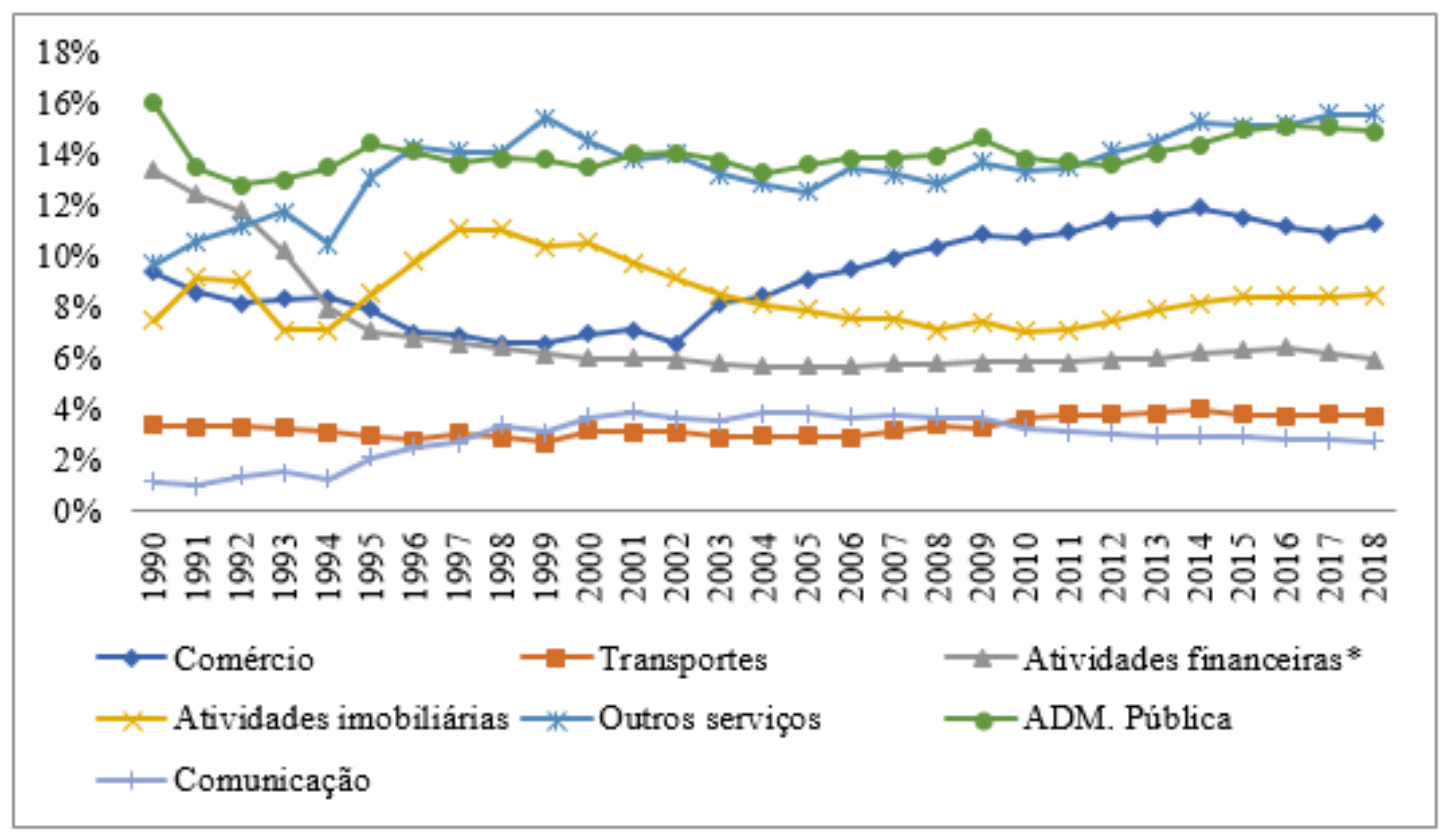

Fonte: Dados do IBGE. Elaboração própria.

*Para a série de atividades financeiras, foi calculada a média móvel devido à inconsistência dos dados entre 1992 e 1994.

De acordo com Rowthorn e Ramaswamy (1998), é natural que em países de renda alta a participação da indústria de transformação no PIB e no emprego se reduza ao longo do tempo, devido a fatores estruturais internos das economias avançadas, como os efeitos combinados do emprego industrial, o rápido crescimento da produtividade na indústria em relação aos serviços, o declínio do preço das manufaturas e a mudança nos padrões de demanda entre os bens 
industriais e serviços. Porém, em economias de rendas média e baixa, a perda dos encadeamentos produtivos gerados pela indústria, em caso de desindustrialização prematura, é algo grave, que pode afetar o processo de crescimento e mudanças estruturais importantes para o desenvolvimento.

A Figura 2 mostra uma queda acentuada da participação da indústria de transformação no PIB. No entanto, o setor permanece sendo o mais importante em termos de encadeamentos produtivos e multiplicadores de produção e emprego, como será mostrado mais à frente. Nesse sentido, a análise das relações de crescimento da produção após a abertura comercial, com a aplicação de métodos clássicos de Insumo-Produto, permite a obtenção de uma visão crítica consistente com cada contexto perpassado pela economia brasileira entre 1990 e 2015.

Figura 2 - Participação dos setores industriais no PIB

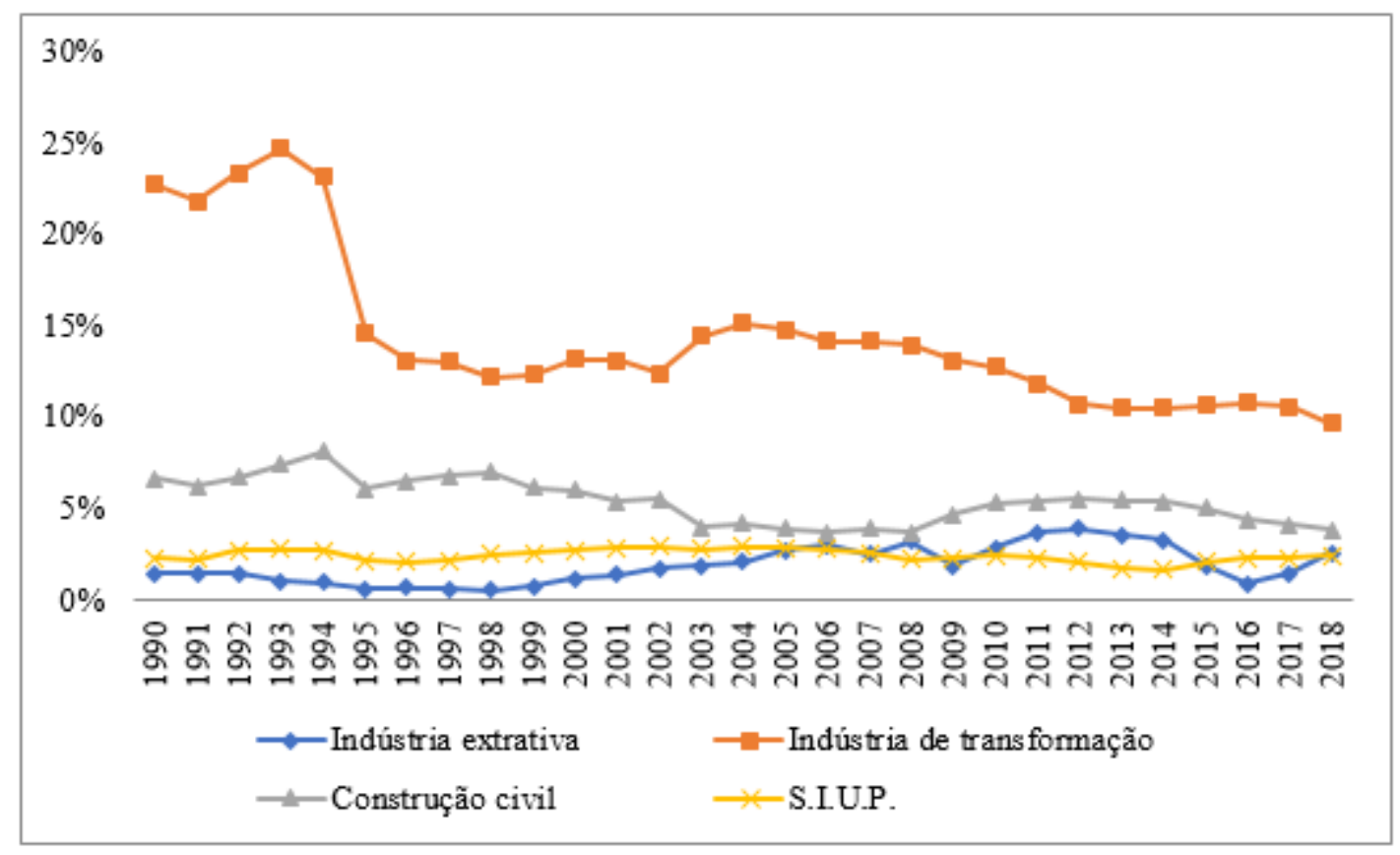

Fonte: Dados do IBGE. Elaboração própria.

A relação descritiva da participação setorial no PIB é importante, pois nos mostra de imediato como se deu o desenvolvimento dos setores, no entanto, supõese que o crescimento ou decréscimo da participação dos setores no PIB não está diretamente relacionado com a geração de encadeamentos ou multiplicadores. Esta foi uma das constatações de Marconi, Rocha e Magacho (2016), ao observarem que apesar do crescimento do setor agrícola na economia brasileira durante os anos 
2000, o setor continuou gerando baixos índices de encadeamentos. Ou, paradoxalmente, no que se refere ao setor manufatureiro que perde participação no PIB, mas continua sendo o setor com maior capacidade de geração de multiplicadores da produção e emprego e maiores conexões intersetoriais.

\section{Base de dados e metodologia}

\subsection{Base de dados}

As MIP's brasileiras são elaboradas pelo Instituto Brasileiro de Geografia e Estatística - IBGE, atualmente, a cada cinco anos. Na presente pesquisa são utilizadas as MIP's quinquenais dos anos de 1990, 1995, 2000, 2005, 2010 e 2015. O Quadro 1 apresenta a estrutura setorial adotada.

\section{Quadro 1 - Estrutura setorial agregada das matrizes de Insumo-Produto}

\begin{tabular}{|l|l|}
\hline Setores & Siglas \\
\hline Agropecuária & S1 \\
\hline Indústrias extrativas & S2 \\
\hline Indústria de transformação & S3 \\
\hline Serviços industriais de utilidade pública & S4 \\
\hline Construção civil & S5 \\
\hline Comércio & S6 \\
\hline Transportes & S7 \\
\hline Informação e comunicação & $\mathrm{S} 8$ \\
\hline Atividades financeiras & $\mathrm{S} 9$ \\
\hline Atividades imobiliárias & $\mathrm{S} 10$ \\
\hline Outras atividades de serviços & $\mathrm{S} 11$ \\
\hline Administração pública & $\mathrm{S} 12$ \\
\hline
\end{tabular}

Fonte: Elaboração própria.

Esta agregação setorial é justificada em razão da compatibilização das matrizes mais recentes com as matrizes da década de 1990 (Sousa Filho et al., 2020). Isso corrobora o objetivo principal do estudo ao apresentar o comportamento dos setores desde a abertura comercial da economia brasileira e seu fraco desempenho sobre o crescimento econômico. O período dos anos 2000 foi marcado por crescimento e maior estabilidade e, finalmente, o período entre 2011 e 2015, novamente foi marcado pelo fraco crescimento. É importante 
destacar que a comparação apenas de forma quinquenal é justificada pela mudança metodológica do IBGE para as matrizes de 2010 e $2015^{1}$.

\subsection{Metodologia}

\subsubsection{Os Índices de Rasmussen/Hirschman}

Os trabalhos de Rasmussen (1956) e Hirschman (1958) nos permite definir as inter-relações entre os setores e o poder que cada setor tem de estabelecer linkages. Desse modo, pôde-se verificar quais setores têm maior poder de encadeamento, possuem ligações para trás (backward effects), capazes de fornecer insumos para demais setores, e ligações para frente (forward effects), capazes de indicar a quantidade de produtos demandada de outros setores da economia pelo setor em questão.

Assim, de acordo com Guilhoto (2011), sendo $b_{i j}$ um elemento da matriz inversa de Leontief $\mathrm{B}, \mathrm{B} *$ sendo a média de todos os elementos de $\mathrm{B}$; e $B_{j}, B_{i}$ sendo a soma de uma coluna e de uma linha qualquer de B, os índices de ligações para trás seriam:

$$
B E=\frac{\left[\frac{B_{j}}{n}\right]}{B^{*}}
$$

Para ter-se os índices de ligação para frente, é necessário definir $F$ como sendo a matriz de coeficientes linha obtida a partir da matriz de consumo intermediário, $G$ a matriz de Ghosh, segundo Miller e Blair (2009), $G=(I-F)^{-1}$, $G^{*}$ a média de todos os elementos de $G$ e $G_{i *}$ a soma de uma linha qualquer de $G$, assim, os índices de ligações para frente seriam:

$$
F E=\frac{\left[\frac{G_{i *}}{n}\right]}{G^{*}}
$$

Os setores podem ser classificados em quatro grupos, dependendo dos seus valores índices: i) setores independentes de outros, se ambos os índices de ligações forem menores que 1; ii) setores dependentes, se ambos os índices são maiores que

\footnotetext{
${ }^{1}$ As matrizes de 2010 e 2015 estão em consonância com as novas recomendações do Sistema de Contas Nacionais da Organização das Nações Unidas de 2008, ao passo que as demais matrizes são referentes às recomendações de 1993.
} 
1, estes são setores que têm um papel importante na economia; iii) setores dependentes na oferta intersetorial, que estimulam a produção em outros setores, se o índice $\mathrm{BE}$ for maior que 1; iv) dependentes na demanda intersetorial, se o índice FE é maior que 1 (Marconi et al., 2016).

\subsubsection{Análise de Encadeamentos Através do Método GHS}

O trabalho de Guilhoto, Sonis e Hewings (2005) apresenta uma decomposição da matriz inversa de Leontief que integra as principais técnicas usadas na estrutura de decomposição de Insumo-Produto e diferencia o impacto de um setor econômico e seus componentes. Os dois métodos tratados foram o enfoque dos setores-chave e o enfoque das ligações puras, este último identificado como as fontes de mudanças na economia e os efeitos internos e externos dos multiplicadores de Miyazawa (1976). Conhecido como método GHS, este é baseado na matriz de coeficientes técnicos $(A)$ :

$$
A=\left[\begin{array}{ll}
A_{j j} & A_{j r} \\
A_{r j} & A_{r r}
\end{array}\right]
$$

$\mathrm{A}_{\mathrm{jj}}$ e $\mathrm{A}_{\mathrm{rr}}$ representam matrizes quadradas de coeficientes técnicos diretos do setor j e do resto da economia, exceto o próprio setor; $A_{\mathrm{jr}}$ e $A_{\mathrm{rj}}$ representam matrizes retangulares dos insumos diretos adquiridos pelo setor $\mathrm{j}$ do resto da economia e os insumos diretos adquiridos pelo resto da economia do setor j. Efetuando a decomposição tripla multiplicativa da matriz inversa de Leontief:

$$
B=(I-A)^{-1}=\left[\begin{array}{cc}
B_{j j} & B_{j r} \\
B_{r j} & B_{r r}
\end{array}\right]=\left[\begin{array}{cc}
\Delta_{j j} & 0 \\
0 & \Delta_{r r}
\end{array}\right]\left[\begin{array}{cc}
\Delta_{j} & 0 \\
0 & \Delta_{r}
\end{array}\right]\left[\begin{array}{cc}
I & A_{j r} \Delta_{r} \\
A_{r j} \Delta_{j} & I
\end{array}\right]
$$

Em que,

$$
\begin{gathered}
D j=(I-A j j)^{-1} \\
D r=(I-A j j)^{-1} \\
D j j=(I-D j A j r D r A r j)^{-1} \\
D r r=(I-D r A r j D j A j r)^{-1}
\end{gathered}
$$


Portanto, a partir do modelo tradicional de Leontief e da formulação apresentada, pode-se derivar indicadores para diversas finalidades. Para o uso do presente estudo, identificou-se como o processo de produção aconteceu na economia e seus desdobramentos em termos de mudanças estruturais, o foco é dado nos índices puros de ligação para trás $(P B E)$ e para frente $(P F E)$ da seguinte forma:

$$
\begin{gathered}
\left(\begin{array}{l}
X_{j} \\
X_{r}
\end{array}\right)=\left(\begin{array}{cc}
\Delta_{j j} & 0 \\
0 & \Delta_{r r}
\end{array}\right)\left(\begin{array}{l}
\Delta_{j} Y_{j}+\Delta_{j} A_{j r} \Delta_{r} Y_{r} \\
\Delta_{r} A_{r j} \Delta_{j} Y_{j}+\Delta_{r} Y_{r}
\end{array}\right) \\
P B E=\operatorname{DrArjDjYj} \\
P F E=\operatorname{DjArjDrYr}
\end{gathered}
$$

O índice $P B E$ mostra o impacto do valor total da produção do setor $j$ no restante da economia, menos a demanda pelos insumos que o setor $j$ produz para si mesmo e o retorno do resto da economia para o setor $j$. O índice PFE indica o impacto do valor total da produção do restante da economia para o setor $j$. O índice total de ligações puras $(T L P)$ para cada setor na economia será:

$$
T L P=P B E+P F E
$$

Os índices de ligações puras são expressos em termos de valor da produção total. Portanto, não é aconselhável realizar uma análise comparativa em diferentes períodos dos índices puros e dos índices de ligações Hirschman-Rasmussen. Para superar tal situação é necessário realizar a normalização dos índices puros, dividindo-se os valores da produção em cada setor pelo valor médio da economia (GUILHOTO et al., 2010). Logo, o índice puro de ligação para trás normalizado torna-se:

$$
P B E N=\frac{\frac{P B E}{\sum_{i}^{n} P B E}}{n}
$$


O índice puro de ligações para frente normalizado dar-se por:

$$
P F E N=\frac{\frac{P F E}{\sum_{i}^{n} P F E}}{n}
$$

Por fim, o índice puro total é:

$$
T L P N=\frac{\frac{T L P}{\sum_{i}^{n} T L P}}{n}
$$

\subsubsection{Multiplicadores da produção e emprego}

Seguindo as recomendações de Miller e Blair (2009), o modelo de InsumoProduto pode ser definido por

$$
x=L f
$$

Onde $x$ é o produto total e $f$ é o vetor de demanda final. A matriz inversa de Leontief é dada por:

$$
L=\left[l_{i j}\right]=(I-A)^{-1}
$$

O multiplicador simples do setor $j$ é especificado por:

$$
m(o)_{j} \sum_{i=1}^{n} l_{i j}
$$

E o multiplicador simples do emprego para o setor $j$ é:

$$
m(h)_{j} \sum_{i=1}^{n} a_{n+1}, i l_{i j}
$$


Onde $a_{n+1}$ é o coeficiente do emprego, i.e., o emprego do setor $j$ dividido pelo produto do setor $j$. É importante destacar que ambos os multiplicadores consideram a demanda das famílias como exógenas.

Cabe destacar que estes métodos são essenciais para o entendimento das relações de Insumo-Produto. Além disso, fornecem indicadores que se relacionam com a capacidade de geração de spillovers, geração de inovação, eficiência e produtividade (Wolff; Nadiri, 1993; Marinova; Phillimore, 2004; Harada, 2015).

A construção dos linkages effects e multiplicadores é realizada por meio de coeficientes técnicos fixos, como aqui demonstrado. Logo, não é necessário realizar um tratamento preliminar dos dados. No entanto, é importante ressaltar que as mudanças metodológicas se constituem em fragilidades em termos comparativos de análise, como já mencionado anteriormente.

\section{Resultados e discussões}

\subsection{Linkage effects setoriais da produção}

As mudanças estruturais que ocorrem no setor manufatureiro envolvem recursos estáticos e dinâmicos que transbordam para diversos outros grupos e setores econômicos. A manufatura cria oportunidades para a acumulação de capital, concentração espacial, economias de aglomeração e economias de escala (Glaeser; Gottlieb, 2009).

Na relação entre crescimento econômico e a atividade industrial, Thirlwall (2002) apresenta três "leis": i) existe uma relação causal forte entre o crescimento do produto da manufatura e o crescimento do PIB; ii) existe uma forte relação causal positiva entre o crescimento do produto da manufatura e o crescimento da produtividade na manufatura como um resultado dos retornos de escala estáticos e dinâmicos (Kaldor-Verdoorn's law); e iii) existe uma relação causal positiva entre a taxa a qual o setor manufatureiro se expande e o crescimento da produtividade além da manufatura, por conta dos retornos decrescentes na agricultura e demais serviços que fornecem trabalho para o setor industrial. Os bens industriais, de forma geral, são internacionalmente transacionáveis, logo, os setores manufatureiros obtêm lucros tanto da demanda doméstica quanto da demanda externa. Quanto maior o crescimento da demanda no comércio externo, maior a geração de círculos virtuosos da produção e maior a competitividade do país no cenário internacional. 
Ao calcular os índices de encadeamentos produtivos para a economia brasileira entre os anos de 2000 e 2009, Marconi, Rocha e Magacho (2016) também relatam tal constatação. Ainda, afirmam que os setores agrícola, mineral e os setores de bens non-tradables possuem pouca capacidade de impulsionar a economia. Basicamente, os índices de backward effects desses setores são baixos, por não terem uma cadeia produtiva grande. Em contraste, de acordo com a pesquisa dos autores, os índices forward effetcs de setores mais modernos e dinâmicos são altos, e estes setores podem estimular o crescimento da produção associados ao desenvolvimento manufatureiro.

Gabriel e Ribeiro (2019) realizaram uma análise robusta sobre o crescimento do setor manufatureiro em 115 países entre 1990 e 2011. Ao aplicar os indicadores de Rasmussen-Hirschman e também o método de campo de influência, os autores constataram que o setor manufatureiro funciona como uma "engrenagem de crescimento" para os países em desenvolvimento. No entanto, o setor vem perdendo participação na economia desses países e gerando menos linkages produtivos.

Assim, aplicando o método de mensuração Rasmussen-Hirschman de linkage effects para os setores produtivos agregados da economia brasileira, foi encontrado uma grande relevância do setor manufatureiro em todos os períodos analisados com a geração de forward effects mais relevantes do que backward effects. No entanto, a relação do tamanho do efeito sobre a economia não evolui com o passar do tempo. O setor de serviços também desempenha um papel importante na geração, principalmente de forward effetcs, mas também apresentando pouco crescimento no decorrer do tempo. Os setores de atividades imobiliárias, administração pública e atividades financeiras foram os que apresentaram os piores resultados, de acordo com a figura 3 . 


\section{Figura 3 - Índices Rasmussen-Hirschman}

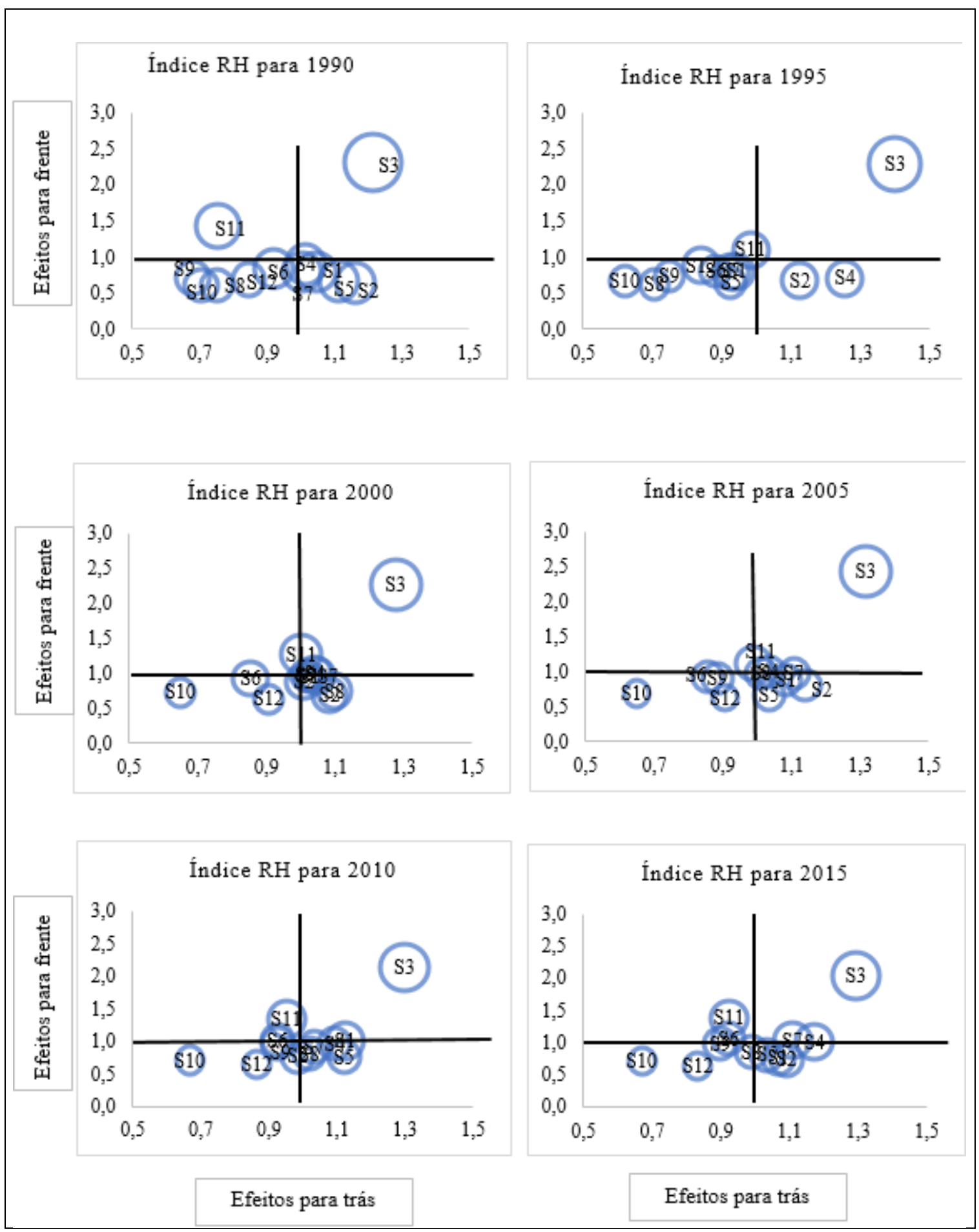

Fonte: Dados das MIP's 1990-2015. Elaboração própria.

Por conseguinte, a análise dos índices de Rasmussen-Hirschman mostrou que os setores da indústria de transformação e o setor de serviços, apesar das perdas, foram os setores com maior capacidade de gerar encadeamentos ao longo 
do período. A abertura e a integração comercial no processo de globalização avançada e as particularidades regionais impuseram mudanças estruturais significativas nos setores da economia. Entre 1990 e 1995 se caracterizava como setor-chave da economia, ou seja, apresentava tanto backward effects quanto forward effects acima da unidade, apenas o setor da indústria de transformação. Em 2000-2005, a indústria de transformação segue como a maior geradora de encadeamentos, porém, já seguida pelo setor de serviços industriais de utilidade pública e pelo setor de serviços. Entre 2005 e 2010 destacaram-se a manufatura, serviços industriais de utilidade pública, transportes, comunicações e serviços. Em 2010 e 2015 os setores da indústria de transformação e serviços de transportes se destacam como setores-chave.

Segundo Dietzenbacher (2000), o setor manufatureiro é o que mais causa efeitos de spillovers. Os efeitos da inovação criados na manufatura podem se propagar por mais setores e aperfeiçoar os processos produtivos. Dessa forma, provocam mudanças estruturais que acumulam efeitos da inovação e determinam a direção dos efeitos e consequências da aplicação tecnológica em cada setor. A mensuração pode ser obtida diretamente pela diagonal dos elementos da matriz inversa de Leontief e pela soma de suas colunas. Para o autor, os efeitos spillovers na inovação são definidos como a mudança na produção total que ocorre em um determinado setor quando ocorre uma mudança inovativa em outro. Os efeitos spillovers podem ainda ser independentes do tamanho da inovação na estrutura específica da demanda final.

O aprofundamento de linkages industriais é algo benéfico por si só, pois equivale a aumentar a especialização e divisão do trabalho na economia e, portanto, a produtividade global. Wolff e Nadiri (1993) afirmam que as relações de investimento em $\mathrm{P} \& \mathrm{D}$, mudanças técnicas e linkages intersetoriais são necessárias para se obter acumulação de capital e aumento significativo da produtividade total dos fatores, sendo que os gastos em $\mathrm{P} \& \mathrm{D}$ demandam a ação conjunta de investimentos privados e públicos, resultando, assim, em benefícios que podem ser ampliados para todos os agentes.

Cabe ressaltar que os índices de HR não consideram o tamanho do setor da economia, e isso se mostra uma fragilidade. Portanto, foram calculados também os índices puros de ligação (GHS) que, em seu cálculo, consideram o valor bruto da produção de cada setor. 
O índice GHS permite uma análise mais aprofundada dos índices de backward effects e forward effects para os setores produtivos da economia como um todo. No que se refere, por exemplo, ao setor de administração pública, para todos os períodos em análise, este setor apresentou índice GHS igual a zero em razão da demanda no consumo intermediário do setor ser igual a zero ${ }^{2}$. No modelo GHS, mais setores se apresentaram como sendo setores-chave da economia em relação ao modelo tradicional de Rasmussen-Hirschman, porém, com o passar do tempo, o poder de encadeamento produtivo decresceu em grande parte deles. O setor de serviços industriais de utilidade pública foi o setor mais relevante no modelo GHS. A indústria de transformação, serviços, construção civil, informação e comunicação, e atividades financeiras também se destacaram, no entanto, com uma volatilidade maior e perda de participação com o passar do tempo, conforme a figura 4.

\footnotetext{
${ }^{2}$ A linha de consumo intermediário do setor de Administração pública, Defesa e seguridade social das MIP's é 0 porque considera-se, de acordo com a metodologia utilizada pelo IBGE, o valor de sua produção igual aos seus custos produtivos.
} 
Figura 4 - Índices GHS

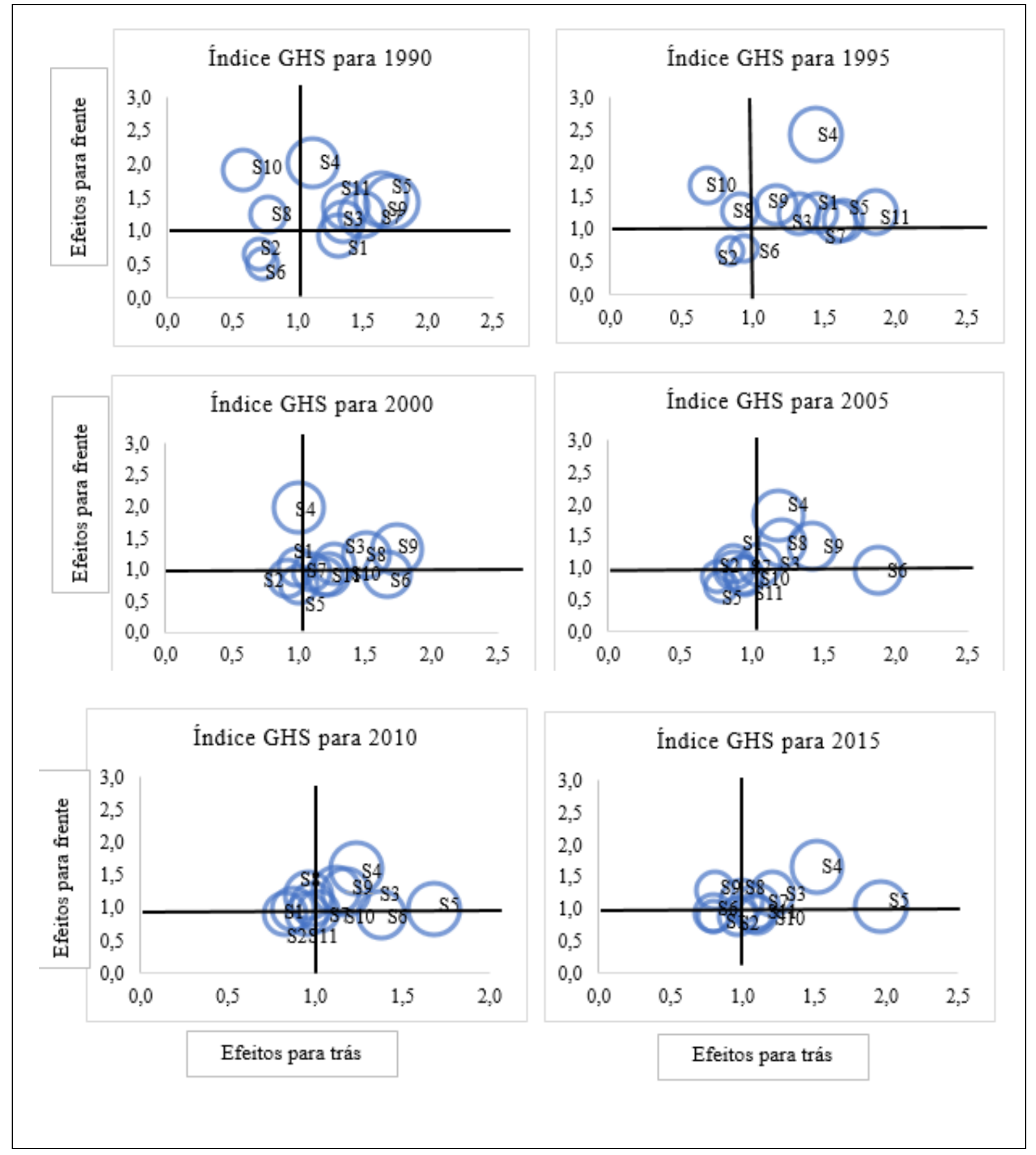

Fonte: Dados das MIP’s 1990-2015. Elaboração própria.

Por fim, os índices GHS totais normalizados para o ano de 1990 mostraram que os setores mais relevantes para a economia do período foram os serviços industriais de utilidade pública, construção civil, transportes, atividades financeiras, e outros serviços. Para o ano de 1995, os serviços industriais de utilidade pública são os mais relevantes, seguindo de outros serviços, construção 
civil, transportes e atividades financeiras. No ano de 2000, os setores que apresentaram mais encadeamentos produtivos foram o comércio, comunicações e instituições financeiras.

Em 2005 destacaram-se os setores de serviços industriais de utilidade pública, comércio, comunicações e atividades financeiras. Em 2010 os três maiores destaques foram a indústria de transformação, serviços industriais de utilidade pública, e a construção civil, sendo que a construção civil foi também o setor mais significativo em 2015, acompanhada dos serviços industriais de utilidade pública e da indústria de transformação, respectivamente, conforme a figura 5.

\section{Figura 5 - Índice de ligações GHS totais}

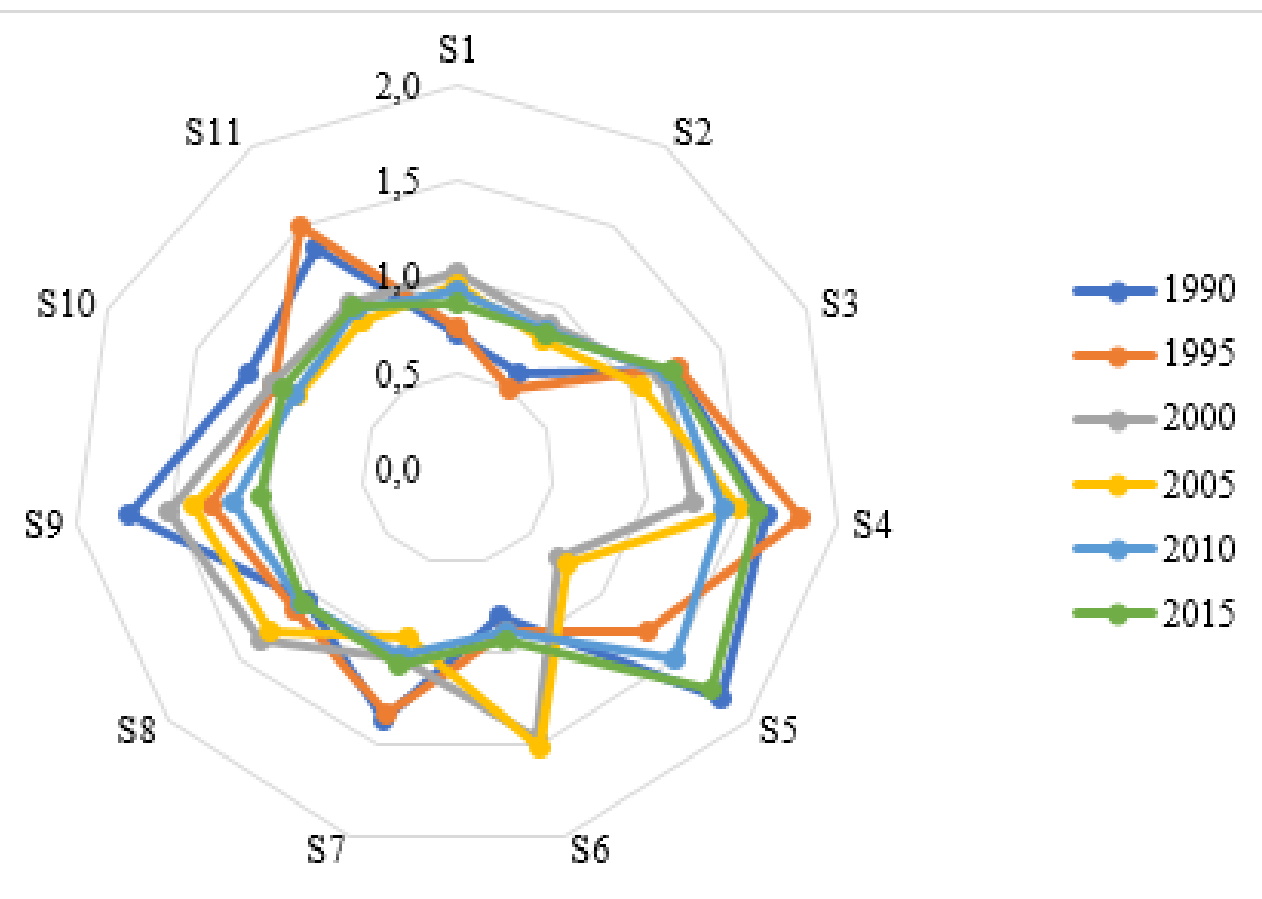

Fonte: Dados das MIP’s 1990-2015. Elaboração própria.

De acordo com Harada (2015), os choques de produtividade e investimentos em P\&D são os principais responsáveis pelas relações de linkages effects. Setoreschave da economia, por meio da especialização vertical, provocam baixos índices de backward effects, porém altos índices de forward effects. Como consequência, esses setores se tornam essenciais para o direcionamento de políticas e surgimento de setores intensivos em tecnologia. $\mathrm{O}$ autor também se volta para as mudanças estruturais que ocorreram na agricultura, um setor historicamente intensivo em 
trabalho, porém, cada vez mais, vem introduzindo maquinarias, produtos químicos e bioquímicos no processo produtivo, devido às mudanças tecnológicas ocorridas na indústria e setores avançados de serviços.

A criação de Pesquisa e Desenvolvimento (P\&D) e, consequentemente, a geração de novas tecnologias, desempenha um papel fundamental para o crescimento setorial no contexto mesoeconômico. Um sistema tecnológico eficiente é o que garante a inovação, competitividade, possibilidade de lucro, concepção de conhecimento tecnológico e mudança tecnológica (Antonelli, 2001). Dessa forma, o processo produtivo que envolve as etapas micro-meso compreende a criação de novos produtos, novos mercados, novas interações organizacionais e, assim, afeta o sistema econômico como um todo.

A mensuração desses efeitos permite determinar quais são os setores-chave da economia, ou seja, aqueles que possuem mais efeitos de spillovers e capacidade de atrair e gerar impactos positivos com complementaridade, economias externas e investimento. Alguns setores, geralmente aqueles mais modernos dentro da manufatura, sempre terão mais possibilidades de crescimento que setores menores ou incapazes de promover investimento induzido. Assim sendo, os mais modernos sempre crescerão mais rápido que os demais, o que corrobora a teoria do crescimento desequilibrado.

\subsection{Multiplicadores da produção e do emprego}

Dietzenbacher (2005) afirma que o tamanho dos setores é relevante para a análise dos multiplicadores. Uma unidade a mais de produção requer, geralmente, mais esforço de uma pequena firma do que de uma grande firma. Um crescimento exógeno pode ocorrer se os gargalos que restringem a produção corrente das firmas forem suprimidos, como a restrição de crédito e trabalho qualificado, por exemplo. $\mathrm{O}$ crescimento da demanda também é um fator importante na análise dos multiplicadores à medida que a oferta pode aumentar para suprir demandas extras levando os produtores a considerar uma expansão produtiva exógena. Portanto, uma alteração exógena na demanda final pode induzir a uma mudança exógena na produção.

De acordo com Gabriel et al. (2020), a geração de multiplicadores setoriais da produção e emprego deve ser mais relevante em economias em desenvolvimento do que em economias desenvolvidas. Isto porque países em 
desenvolvimento possuem maior atraso tecnológico, logo, multiplicadores da produção e emprego mais elevados nessas economias são importantes para aumentar o PIB per capita. Essencialmente, o setor manufatureiro de bens transacionáveis possui grande relevância para o crescimento dessas economias no longo prazo.

Pereira, Bastos e Perobelli (2013) analisaram os multiplicadores do emprego e da produção dos setores de serviços para o Brasil utilizando a MIP de 2005. Para os autores, os setores de serviços da economia brasileira possuem alta capacidade de criação de empregos, no entanto, baixa capacidade de geração de produto. Os resultados apresentados pela pesquisa não apontaram nenhum setor de serviços considerado como "setor-chave" para a economia. Assim, apresentaram pouca capacidade de geração de encadeamentos para os demais setores. No que se refere aos setores industriais, os autores afirmam que estes mostraram-se muito mais relevantes para o nível de multiplicadores da produção e geração de linkage effects.

No que se refere aos resultados aqui apresentados, na comparação entre os anos de 1990 e 1995, os setores que se destacaram em termos de elevação dos multiplicadores foram: serviços industriais de utilidade pública, serviços de intermediação financeira, administração pública e outros serviços. Os demais setores apresentaram queda do multiplicador, sendo as quedas mais acentuadas nos setores da agropecuária, indústria de transformação e construção civil. Em 1995 e 2000, a relação dos resultados se diversificou um pouco mais, sendo que os setores que apresentaram ganhos foram os setores agropecuário, construção civil, transportes, comunicações, instituições financeiras e administração pública. A indústria de transformação, serviços industriais de utilidade pública e outros serviços apresentaram perdas mais acentuadas, de modo geral.

Para os anos 2000 e 2005, os setores da agropecuária, extração mineral, indústria de transformação e transportes tiveram ganhos mais relevantes. A maior perda deu-se em serviços financeiros. Os setores de serviços de utilidade pública, construção civil, comércio, comunicações, atividades imobiliárias, outros serviços e administração pública apresentaram ligeiros ganhos. Entre 2005-2010 há queda acentuada dos multiplicadores da agropecuária, extração mineral e outros serviços, assim como, em menor grau, na indústria de transformação. Apenas quatro setores aumentaram de forma mais relevante seus multiplicadores, sendo estes a construção civil, comércio, serviços financeiros e serviços industriais de utilidade 
pública. Por fim, em 2010 e 2015 grande parte dos setores tiveram perda ou pouco se alterou o valor dos multiplicadores setoriais. Verificou-se ganhos mais significativos apenas nos setores agropecuária, indústria extrativa e serviços industriais de utilidade pública, conforme a figura 6 .

\section{Figura 6 - Multiplicadores totais da produção}

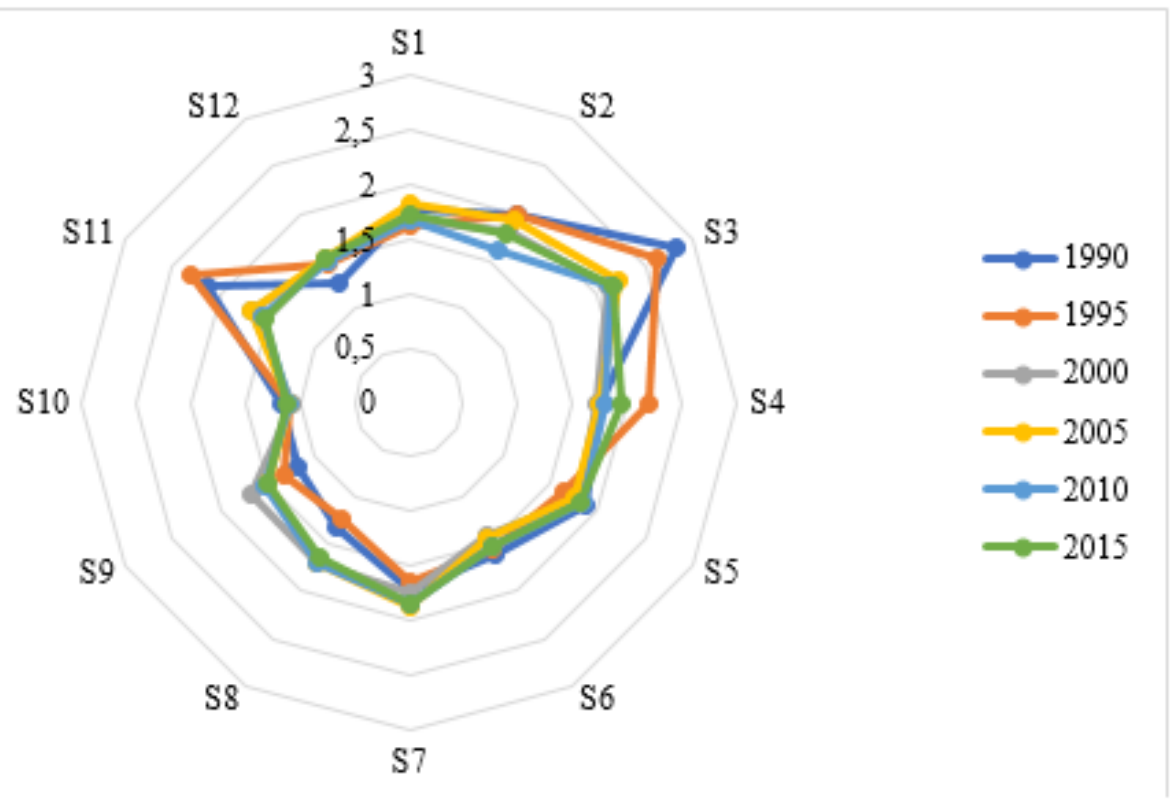

Fonte: Dados das MIP's 1990-2015. Elaboração própria.

A análise dos multiplicadores setoriais apresenta uma tendência conjuntural de crescimento ou recessão do produto dada a estratégia de crescimento adotada pelo setor. Como a análise foi efetuada de forma agregada, a eficiência é marcante em todos os setores, de maneira geral, os setores apresentam-se interligados a uma tecnologia média de produção empregada em economias emergentes. Pode-se destacar, portanto, que o incremento da demanda e estabilidade econômica são fatores-chave para a geração e aumento dos multiplicadores.

Assim como os multiplicadores da produção, os multiplicadores simples do emprego $^{3}$ setorial mensuram a capacidade de geração de empregos dado um aumento de $\mathrm{R} \$ 1$ milhão de reais na demanda final ${ }^{4}$. A importância do fator trabalho para os macrossetores produtivos é também destacada por Long e Plosser

\footnotetext{
${ }^{3}$ Há a possibilidade de se calcular multiplicadores de emprego do tipo 1, do tipo 2 e totais. Para saber mais, ver Miller e Blair (2009).

${ }^{4} \mathrm{~A}$ unidade monetária de milhão de reais refere-se à mensuração das matrizes em $\mathrm{R} \$$ milhões correntes.
} 
(1983) como elemento de encadeamento de choques na produção. No entanto, a interdependência da estrutura de Insumo-Produto é limitada para captar os choques nas flutuações agregadas da produção. Conley e Dupor (2003) reforçam a modelagem multissetorial da estrutura de Insumo-Produto por meio de modelos econométricos espaciais com a finalidade de mensurar a produtividade dos fatores de produção e concluem que setores complementares que compartilham os mesmos fornecedores de insumos intermediários têm maior capacidade de ampliar seus níveis de produtividade e multiplicadores produtivos.

Notadamente, pode-se perceber a grande perda da capacidade de criação de empregos nos setores produtivos da economia brasileira. Até o ano de 2000, a agropecuária gerava mais de 500 empregos diretos e indiretos para cada $\mathrm{R} \$ 1$ milhão de variação na sua demanda final. A partir de 2005, com a introdução de máquinas e equipamentos tecnológicos na produção, o avanço do agronegócio e, portanto, a mecanização da produção em larga escala substituiu o fator trabalho. Em 2015, a cada R\$ 1 milhão de variação na demanda final do setor, em torno de 74 empregos seriam criados. Assim, como na agropecuária, a mesma análise pode ser transposta para grande parte dos setores, com destaque para o comércio, e o setor de outros serviços, conforme a figura 7.

Figura 7 - Multiplicadores setoriais do emprego (1990-2015)

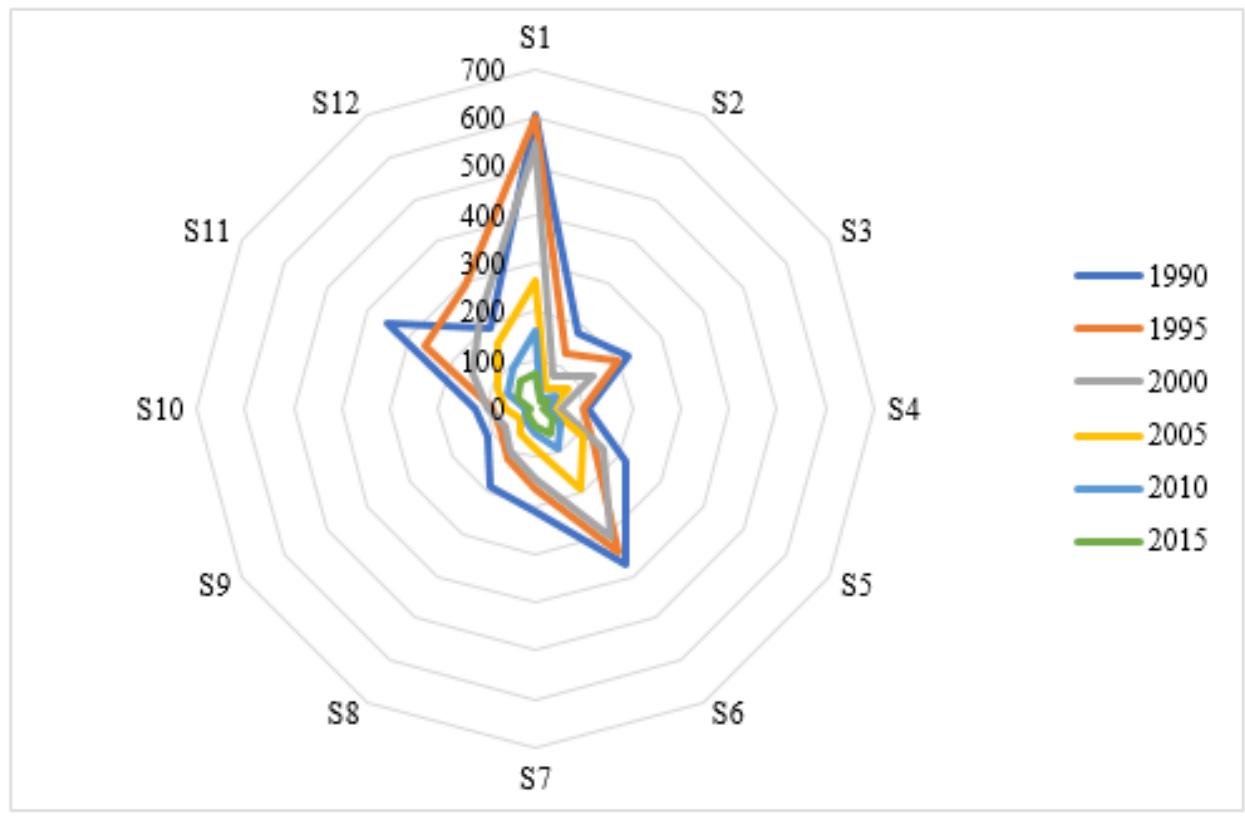

Fonte: Dados das MIP's 1990-2015. Elaboração própria. 
A tendência decrescente da geração de multiplicadores setoriais do emprego deve ser vista com preocupação, pois, apesar do incremento tecnológico na produção, é interessante que o emprego siga uma trajetória que acompanhe o desenvolvimento de novas tecnologias e seja capaz de se adaptar a novos fatores econômicos. A criação de empregos setoriais pode ser vista como um proxy do aquecimento da atividade e do desenvolvimento setorial (Pyka; Saviotti, 2011). Mesmo em setores intensivos em tecnologia e com elevada produtividade, a criação de emprego é um dos fatores preponderantes para o estímulo ao desenvolvimento no longo prazo. Basicamente, novos setores industriais e de serviços avançados são os mais beneficiados por esse sistema dinâmico de emergência de conhecimentos e inovações gerados pelo trabalho qualificado.

Por fim, qualquer comparativo em termos de magnitude dos indicadores aqui apresentados, com relação a outros artigos publicados, deve ser feito com cautela em função dos períodos, metodologias de mensuração das MIP's e nível de agregação setorial. No entanto, é interessante citar alguns trabalhos que apresentam resultados similares utilizando os mesmos métodos. De forma geral, Hilgemberg e Guilhoto (2004) calcularam os linkage effects e multiplicadores totais e do emprego para a economia brasileira entre 1990 e 1999, em sete macrossetores. Os resultados diferem dos daqui apresentados em termos de magnitude, porém mostram a tendência de redução dos multiplicadores do emprego e a estagnação dos linkages produtivos devido ao aumento da dependência da produção nacional em relação a insumos importados.

Cardoso, Domingues e Britto (2015) apresentaram multiplicadores da produção setorial, apenas para o ano de 2005, bem próximos em termos de magnitude aos aqui mostrados. Marconi, Rocha e Magacho (2016) calcularam os multiplicadores da produção, os índices de Rasmussen-Hirschman e o índice GHS para 18 setores entre 2000 e 2009. Os autores utilizaram a metodologia de Guilhoto e Sesso Filho (2005) para a estimação da MIP, contudo, apesar de diferir da estimação oficial do IBGE, os resultados para 2000 e 2005 também corroboram o cálculo apresentado no presente artigo. Outros trabalhos como Matias (2006); Souza (2010); Pereira, Bastos e Perobelli (2013) trazem elementos relevantes para a análise de Insumo-Produto e corroboram as discussões sobre crescimento econômico setorial e desenvolvimento produtivo. 


\section{Considerações finais}

A desaceleração econômica que ocorreu no Brasil desde a década de 1980 causou no país uma redução estrutural de crescimento, queda de produtividade, volatilidade nas taxas de crescimento do PIB e redução da tendência de crescimento no longo prazo. O Brasil passou por significativas reformas estruturais entre 1990 a 2000, que contribuíram para a estabilidade econômica da década seguinte. No entanto, a crise financeira de 2008 fragilizou as condições externas da economia nacional, que apresentou significativos déficits estruturais que levaram o país a sofrer perdas e uma prolongada recessão posteriormente. A análise dos índices de encadeamentos produtivos mostrou como os setores reagiram às conjunturas econômicas dos períodos em estudo, mesmo considerando que os empecilhos de ordem estrutural se mantiveram ao longo do tempo e ainda marcam a economia brasileira profundamente. De um modo geral, as questões referentes à baixa qualificação da força de trabalho, burocratização exacerbada do Estado, carga tributária elevada, dentre outros, contribuem para que não houvesse incorporação tecnológica e produtiva na economia brasileira.

Os índices Rasmussen-Hirschman pouco se alteraram no período analisado, permanecendo uma espécie de homogeneidade e estagnação entre os setores, com exceções na indústria de transformação e outros serviços. Ao comparar com os resultados do índice GHS normalizado, pode-se observar os efeitos conjunturais sobre os setores de uma forma mais heterogênea, especificamente, os setores da agropecuária, extração mineral, indústria de transformação e comércio ficaram estagnados em 1990 e 1995. Os serviços industriais de utilidade pública, construção civil, transportes, instituições financeiras e outros serviços foram os que mais se destacaram. Nos anos 2000 e 2005 foram comércio, comunicações e atividades financeiras, sendo que em 2005 os serviços industriais de utilidade pública cresceram novamente, após queda em 2000. Entre 2010 e 2015, os maiores destaques foram a indústria de transformação, serviços industriais de utilidade pública e construção civil.

Os multiplicadores setoriais mostraram desempenho mais forte para os setores de indústria de transformação, outros serviços e serviços de transportes. De forma geral, a indústria de transformação manteve um multiplicador acima de 2 em todos os períodos analisados. Demonstrando que, mesmo em períodos recessivos como em 2010-2015, sua capacidade de geração de efeitos 
multiplicadores sobre a economia se manteve. No entanto, cabe ressaltar que dentro deste setor há heterogeneidade entre os subsetores industriais, a qual não é captada na análise, em decorrência do elevado grau de agregação setorial e, portanto, constitui-se como uma importante limitação.

A redução da magnitude dos multiplicadores setoriais do emprego ao longo do período analisado pode refletir, em parte, o incremento tecnológico na produção. No entanto, dado o nível de desenvolvimento produtivo brasileiro, pautado, essencialmente, em produtos de média, baixa-média tecnologias e commodities, é importante destacar que a economia pode não ter feito a transição para a criação de empregos tecnológicos, bem como a qualificação da mão de obra voltada para a suprir as alterações na demanda e comércio que levaram ao processo de mudanças estruturais nos fatores capital e trabalho, assim como, a agregação de valor em insumos intermediários.

Por conseguinte, o investimento na industrialização continua sendo um fator importante para o desenvolvimento das economias, principalmente em países em desenvolvimento e emergentes no século XXI, como o Brasil. O aumento da capacidade de criação de linkage effects e dos multiplicadores da produção setorial é importante, pois o crescimento da produção manufatureira promove a criação de empregos formais e contribui para a elevação dos salários, da demanda agregada, do consumo doméstico, da formação de encadeamentos produtivos complexos, etc. (Jones, 2007). De acordo com Dopfer, Foster e Potts (2004), o processo de crescimento mesoeconômico deriva destes elementos e é essencial para a manutenção de novas dinâmicas econômicas que envolvem o processo de inovação. Além disso, também acarreta organizações de ideias e conhecimentos sobre adaptação das novas estruturas em ordem de estabilizar o sistema econômico. Assim, o domínio organizacional microeconômico repercute em novas capacidades dos agentes no processo de maximização de seu bem-estar. Nesse sentido, a análise da evolução dos multiplicadores e encadeamentos setoriais de Insumo-Produto continua fornecendo importantes elementos de diagnóstico e proposição de políticas para a economia brasileira. 


\section{Referências}

ANTONELLI, C. The microeconomics of technological systems. Oxford: Oxford University Press, 2001.

CARDOSO, D.; DOMINGUES, E.; BRITTO, G. Interconexões entre estrutura produtiva, fluxo de renda e consumo na economia brasileira: uma aplicação de uma matriz de contabilidade social e financeira (MCS-F) com abertura das famílias. IN: Encontro Nacional de Economia, 43, 2015, Florianópolis.

CONLEY, T.; DUPOR, B. A spatial analysis of sectoral complementarity. Journal of Political Economy, v. 111, n. 2, p. 311-352, 2003.

DIETZENBACHER, E. The measurement of interindustry linkages in the Nerherlands. Economic Modelling, v. 9, n. 4, p. 419-437, 1992.

DIETZENBACHER, E. Spillovers of innovation effects. Journal of Policy Modeling, v. 22, n. 1, p. 27-42, 2000.

DIETZENBACHER, E. More on multipliers. Journal of Regional Science, v. 45, n. 2. p. 421-426, 2005.

DIETZENBACHER, E.; LUNA, I.; BOSMA, N. Using average propagation lengths to identify production chains in the Andalusian economy. Estudios de Economia Aplicada, v. 23, n. 2, p. 405-422, 2005.

DOPFER, K.; FOSTER, J.; POTTS, J. Micro-meso-macro. Journal of Evolutionary Economics, v. 14, p. 263-279, 2004.

GABRIEL, L.; RIBEIRO, L. Economic growth and manufacturing: an analysis using panel VAR and intersectoral linkages. Structural Change and Economic Dynamics, v. 49, p. 43-61, 2019.

GABRIEL, L.; RIBEIRO, L.; JAYME JUNIOR, F.; OREIRO, J. L. Manufacturing, economic growth, and real Exchange rate: empirical evidence in panel data and input-output multipliers. PSL Quarterly Review, v. 73, p. 51-75, 2020.

GIAMBIAGI, F.; MOREIRA, M. A economia brasileira nos anos 90. Rio de Janeiro: BNDES, 1999.

GLAESER, E.; GOTTLIEB, J. The wealth of cities: agglomeration economies and spatial equilibrium in the United States. NBER Working Paper Series, p. 1-77. Cambridge, 2009. 
GUILHOTO, J.; PICERNO, A. Estrutura produtiva, setores-chave e multiplicadores setoriais: Brasil e Uruguai comparados. Revista Brasileira de Economia, v. 49, n. 1, p. 35-61, 1995.

GUILHOTO, J.; SONIS, M.; HEWINGS, J. Linkages and multipliers in a multiregional framework: integration of alternative approaches. MPRA Paper, $n$. 38213 , p. 1-21, 2005.

GUILHOTO, J.; SESSO FILHO, U. Estimação da matriz insumo-produto a partir de dados preliminares das contas nacionais. Economia Aplicada, v. 9, n. 18, p. 277 $299,2005$.

GUILHOTO, J.; AZZONI, C.; ICHIHARA, S.; KADOTA, D.; HADDAD, E. Matriz de Insumo-Produto do Nordeste e Estados: metodologia e resultados. Fortaleza: Banco do Nordeste, 2010.

GUILHOTO, J. Input-output analysis: theory and foundations. São Paulo: Universidade de São Paulo, 2011.

HARADA, T. Changing productive relations, linkage effects, and industrialization. Economic Systems Research, v. 27, n. 3, p. 374-390, 2015.

HILGEMBERG, T.; GUILHOTO, J. Economic opening and its effects on the Brazilian labour market on the decade of 1990. MPRA Paper n. 54015, p. 1-34, 2004.

HIRSCHMAN, A. The strategy of economic development. Yale: Yale University Press, 1958.

INSTITUTO BRASILEIRO DE GEOGRAFIA E ESTATÍSTICA. Contas nacionais: matrizes de Insumo-Produto. Disponível em: https://www.ibge.gov.br/estatisticas/economicas/contas-nacionais/9085-matrizde-Insumo-Produto.html?=\&t=downloads. Acesso em: 12 nov. 2020.

JONES, C. The weak link theory of economic development. Hong Kong Institute for Monetary Research Working paper n. 4, 2007.

LONG, J.; PLOSSER, C. Real business cycles. Journal of Political Economy, v. 91, n. 1, p. 39-69, 1983.

MARCONI, N.; ROCHA, I.; MAGACHO, G. Sectorial capabilities and productive structure: an input-output analysis of the key sectors of the Brazilian economy. Brazilian Journal of Political Economy, v. 36, n. 3, p. 470-492, 2016. 
MARINOVA, D.; PHILLIMORE, J. Models of Innovation. In: SHAVININA, L. (Ed.). The International Handbook on Innovation. Amsterdam: Elsevier, 2004.

MATIAS, A. Análise da evolução estrutural do setor de serviços no Brasil: uma abordagem de Insumo-Produto. Dissertação (Mestrado em Economia) Universidade Estadual de Maringá. Programa de Mestrado em Economia, Maringá, 2006.

MILLER, R.; BLAIR, P. Input-output analysis: foundations and extensions. Cambridge: Cambridge University Press, 2009.

MIYAZAWA, K. Input-Output analysis and the structure of income distribution. New York: Springer, 1976.

MOREIRA, G; ALMEIDA, L.; GUILHOTO, J.; AZZONI, C. Productive structure and income distribution: the Brazilian case. The Quarterly Review of Economics and Finance, v. 48, p. 320-332, 2008.

PASINETTI, L. Structural economic dynamic: a theory of the economic consequences of human learning. Cambridge: Cambridge University Press, 1993.

PEREIRA, M.; BASTOS, S.; PEROBELli, F. Análise sistêmica do setor de serviços no Brasil para o ano de 2005. Pesquisa e Planejamento Econômico, v. 43, n. 1.p. 161-201, 2013.

PYKA, A.; SAVIOTTI, P. Economic growth through the emergence of new sectors. IN: MANN, S. (Ed.). Sectors Matter! Exploring Mesoeconomics. Londres, Springer, 2011.

RASMUSSEN, N. Studies in inter-sectoral relations. Amsterdam: North Holland, 1956.

RODRIGUES, K.; SESSO FILHO, U.; BRENE, P.; CÂMARA, M. Estrutura produtiva de Porto Alegre/RS: estimativa dos multiplicadores de produção, emprego e rendimentos para 2008. Revista Brasileira de Estudos Regionais e Urbanos, v. 10, n. 2, p. 234-252, 2016.

ROWTHORN, B.; RAMASWAMY, R. Growth, Trade, and deindustrialization. IMF Working Paper, p. 1-28, 1998.

SOUSA FILHO, J.; SANTOS, G.; RIBEIRO, L. Structural changes in the Brazilian Economy 1990-2015. Economic Systems Research, p. 1-22, 2020. 
SOUZA, K. As múltiplas tendências da terciarização: uma análise de InsumoProduto da expansão do setor de serviços. Dissertação (Mestrado em Economia) - Universidade Federal de Juiz de Fora, Juiz de Fora, 2010.

THIRLWALL, A. The nature of economic growth: an alternative framework for understanding the performance of nations. Northampton: Edward Elgar, 2002.

TIMMER, M.; LOS, B.; STEHRER, R.; DE VRIES, G. Fragmentation, incomes and jobs: an analysis of European competitiveness. In: Panel Meeting of Economic Policy, 57, 2012.

WOLFF, E.; NADIRI, M. Spillover effects, linkage structure, and research and development. Structural Change and Economic Dynamics, v. 4, n. 2, p. 315-330, 1993. 